\title{
Desempenho produtivo de ovinos em confinamento alimentados com farelo de manga
}

\author{
[Productive performance of confined sheep fed mango meal] \\ L.G.R. Pereira ${ }^{1}$, A.L.S. Aragão ${ }^{2}$, R.D. Santos $^{3}$, J.A.G. Azevêdo ${ }^{4}$, A.L.A. Neves ${ }^{1}$, \\ A.L. Ferreira ${ }^{5}$, M.L. Chizzotti $^{6}$ \\ ${ }^{1}$ Embrapa Gado de Leite - Juiz de Fora, MG \\ ${ }^{2}$ Aluno de pós-graduação - Universidade Federal do Vale do São Francisco - Petrolina, PE \\ ${ }^{3}$ Embrapa Semiárido - Petrolina, PE \\ ${ }^{4}$ Universidade Estadual de Santa Cruz - Ilhéus, BA \\ ${ }^{5}$ Universidade Federal de Minas Gerais - Belo Horizonte, MG \\ ${ }^{6}$ Universidade Federal de Lavras - Lavras, MG
}

\begin{abstract}
RESUMO
Avaliou-se o efeito da inclusão do farelo de manga em substituição ao milho moído em dietas para ovinos sobre o consumo de nutrientes e de frações fibrosas, o ganho de peso e a conversão alimentar. Estudou-se também o benefício líquido e a taxa de retorno do custo total da dieta. Foram utilizados 24 cordeiros Santa Inês, distribuídos em delineamento inteiramente ao acaso, com quatro tratamentos, constituídos pelas porcentagens de inclusão do farelo de manga, em substituição ao milho, de 0, 33, 66 e 100\%, e seis repetições. Não houve efeito da inclusão do farelo de manga sobre o consumo de nutrientes e o ganho de peso dos animais. Verificou-se efeito quadrático $(\mathrm{P}<0,05)$ da porcentagem de inclusão do farelo de manga sobre a conversão alimentar. O farelo de manga pode substituir o milho em dietas para ovinos sem comprometer o consumo e o ganho de peso e reduz o custo da alimentação, o que sugere ser ele um alimento alternativo para ovinos em confinamento.
\end{abstract}

Palavras-chave: ovino, consumo, ganho de peso, benefício líquido

\begin{abstract}
The effect of inclusion of mango meal to replace corn in sheep diets was evaluated. The variables evaluated were the consumption of nutrients and fibre fractions, weight gain and feed conversion. In addition, a study of the net benefit and rate of return on the total cost of the diets was conducted. Twentyfour lambs Santa Inês, distributed in a completely randomized design with four treatments, consisting of the inclusion levels of mango meal replacing corn (0,33, 66 and 100\%) and six repetitions. There was no effect of the inclusion levels of mango meal on nutrients intake and weight gain of animals. There was a quadratic effect $(P<0.05)$ of inclusion levels of mango meal on feed conversion. The mango meal can replace corn in diets without compromising intake and weight gain, while reducing the cost of feed, indicating the possibility of alternative use as feed for confined sheep.
\end{abstract}

Keywords: sheep, intake, weight gain, net benefit

\section{INTRODUÇÃO}

O sertão do Vale do São Francisco abriga efetivos de ovinos e caprinos que ocupam as primeiras posições no contexto nacional. $\mathrm{Na}$ região de sequeiro, a caprinovinocultura é a

Recebido em 1 de dezembro de 2011

Aceito em 9 de janeiro de 2013

E-mail: luiz.gustavo@cnpgl.embrapa.br atividade de maior importância econômicosocial, uma vez que a pecuária, quando comparada à agricultura, é menos afetada pela seca e predomina sobre esta última em quase todas as regiões semiáridas do mundo. Embora possua um rebanho numericamente expressivo, a criação e terminação de ovinos, exclusivamente 
em pasto, praticada na maioria das propriedades rurais do semiárido nordestino, tem se mostrado ineficaz, pois este processo está submetido à irregularidade na disponibilidade de forragem da caatinga, tendo como consequência o predomínio de baixos índices zootécnicos, abates tardios, qualidade de carne incompatível com a demanda e irregularidade na oferta de produtos cárneos.

O confinamento de ovinos tem recebido, nos últimos anos, crescente adoção em virtude da redução do tempo para o abate, da maior eficiência no controle sanitário, da melhor qualidade da carcaça, da manutenção da oferta de forragem no período de escassez e, consequentemente, de uma regularidade maior na produção e na qualidade da carne ovina disponibilizada no mercado (Lage et al., 2010).

Apesar de todos os aspectos positivos apresentados pelo confinamento, nem sempre esse sistema de produção é economicamente viável, uma vez que sua viabilidade está atrelada às oscilações de preço dos grãos, principalmente milho e soja. Uma possibilidade de redução dos efeitos dessa situação sazonal é reduzir o custo das dietas empregadas nesse sistema de produção, por meio do aproveitamento de fontes de alimentos disponíveis em cada região (Lage et al., 2010). Os coprodutos da fruticultura são apontados como opção alimentar para ruminantes (Azevêdo et al., 2012).

O submédio do Vale do São Francisco, em especial o polo Juazeiro/Petrolina, é uma das principais regiões produtoras de frutas do Brasil. Entre as frutíferas cultivadas nessa região, destaca-se a manga, com 375.800 toneladas produzidas no ano de 2009 (IBGE, 2011).

Frequentemente, nos períodos de safra, o preço da manga atinge valores muito baixos, e os produtores muitas vezes optam pela não comercialização dessa fruta, para evitar custos com colheita e transporte; assim, a utilização desses frutos na alimentação de ruminantes pode ser uma estratégia para a obtenção de produtos nobres, como a carne e o leite, além de uma opção para o aumento da renda dos produtores dessa região.
Nesse contexto, objetivou-se avaliar o desempenho produtivo de ovinos Santa Inês em confinamento, submetidos a porcentagens crescentes de farelo do fruto da manga, em substituição ao milho moído, bem como a viabilidade econômica dessa substituição.

\section{MATERIAL E MÉTODOS}

O experimento foi realizado no Campus de Ciências Agrárias da Universidade Federal do Vale do São Francisco, localizado no município de Petrolina, PE. Os tratamentos constituíram-se de: capim-elefante $(\mathrm{CE})+$ concentrado padrão (farelo de soja + milho); $\mathrm{CE}+33 \%$ de inclusão do farelo de manga (FM) em substituição ao milho do concentrado padrão; $\mathrm{CE}+66 \%$ de inclusão do FM em substituição ao milho do concentrado padrão e $\mathrm{CE}+100 \%$ de inclusão do FM em substituição ao milho (Tab. 1). O farelo de manga foi obtido de frutas integrais sem valor comercial, que foram desintegradas em picadeira estacionária, desidratadas ao sol por 48 horas e depois moídas em partículas de $5 \mathrm{~mm}$.

Foram utilizados 24 ovinos machos castrados, da raça Santa Inês, com peso vivo médio inicial de $23,3 \pm 2,5 \mathrm{~kg}$. Inicialmente, os animais foram pesados, vermifugados e, em seguida, alojados em baias individuais providas de comedouro, bebedouro e saleiro.

Os animais foram alimentados à vontade, duas vezes por dia, às 7h30min e às $16 \mathrm{~h} 30 \mathrm{~min}$, com rações formuladas para serem isonitrogenadas $12 \%$ PB na matéria seca total -, sendo a relação concentrado:volumoso fixada em 60:40 (Tab. 1). As quantidades fornecidas foram ajustadas para que as sobras correspondessem a $15 \%$ do oferecido. Água e mistura mineral foram oferecidas à vontade.

O experimento teve duração de 85 dias, sendo 14 dias de adaptação dos animais às baias e ao manejo e 75 dias de coleta de dados. Durante o período de coleta, foi quantificado diariamente o alimento fornecido aos animais e as sobras no cocho, obtendo-se pela diferença entre oferecido e sobra o valor de consumo. As pesagens dos animais foram realizadas quinzenalmente, no início da manhã, antes da primeira refeição. 
Desempenho produtivo de ovinos...

Tabela 1. Composição percentual dos ingredientes nas dietas (\% na MS)

\begin{tabular}{lcccc}
\hline \multirow{2}{*}{ Ingrediente } & \multicolumn{4}{c}{ Inclusão de farelo de manga (\%) } \\
\cline { 2 - 5 } & 0 & 33 & 66 & 100 \\
\hline Capim-elefante & 40 & 40 & 40 & 40 \\
Milho & 46,9 & 31,3 & 16 & - \\
Farelo de manga & - & 15,2 & 30,2 & 45,7 \\
Ureia & - & 0,4 & 0,7 & 1,1 \\
Farelo de soja & 13,1 & 13,1 & 13,1 & 13,2 \\
\hline Total & 100 & 100 & 100 & 100 \\
\hline
\end{tabular}

As amostras dos alimentos fornecidos e das sobras foram avaliadas em duplicatas, sendo determinados os teores de matéria seca (MS), matéria orgânica $(\mathrm{MO})$, proteína bruta $(\mathrm{PB})$, energia bruta $(\mathrm{EB})$, extrato etéreo $(\mathrm{EE})$ segundo as metodologias descritas por Silva e Queiroz (2002), e fibra em detergente neutro (FDN) e fibra em detergente ácido (FDA) segundo Van Soest et al. (1991). Os teores de carboidratos totais (CT) e carboidratos não fibrosos (CNF) foram obtidos pelas equações: $\mathrm{CT}=100-(\mathrm{PB} \%$ $+\mathrm{EE} \%+\mathrm{MM} \%)$ e $\mathrm{CNF}=100-(\mathrm{PB} \%+\mathrm{EE} \%+$ $\mathrm{MM} \%+\mathrm{FDN} \%)$, de acordo com Hall (2000).

As análises para determinação da energia bruta foram realizadas por meio de bomba calorimétrica adiabática marca PARR (AOAC, 1980). A composição bromatológica do capimelefante, do farelo de manga e das rações totais está descrita na Tab. 2.

Tabela 2. Composição bromatológica do capim-elefante (CE), do farelo de manga (FM) e das rações totais contendo diferentes porcentagens de farelo de manga em substituição ao milho moído

\begin{tabular}{lcccccc}
\hline \multirow{2}{*}{ Variável } & \multirow{2}{*}{$\mathrm{FE}$} & \multicolumn{4}{c}{ Inclusão de farelo de manga (\%) } \\
\cline { 4 - 7 } & & & 0 & 33 & 66 & 100 \\
\hline $\mathrm{MS}^{1}$ & 23,51 & 89,53 & 62,95 & 63,1 & 63,24 & 63,39 \\
$\mathrm{MO}^{2}$ & 91,7 & 96,35 & 94,5 & 94,35 & 94,19 & 94,03 \\
$\mathrm{MM}^{2}$ & 8,31 & 3,65 & 5,5 & 5,65 & 5,81 & 5,97 \\
$\mathrm{~PB}^{2}$ & 4,66 & 4,47 & 11,69 & 12,06 & 12,17 & 12,51 \\
$\mathrm{FDN}^{2}$ & 78,35 & 22,86 & 44,15 & 44,67 & 45,17 & 45,71 \\
$\mathrm{FDA}^{2}$ & 51,87 & 15,3 & 27,18 & 28,54 & 29,06 & 31,27 \\
$\mathrm{EE}^{2}$ & 1,9 & 5,12 & 2,96 & 3,3 & 3,63 & 3,98 \\
$\mathrm{~EB}^{3}$ & 2962,08 & 4456,28 & 3636,28 & 3740,76 & 3843,23 & 3951,08 \\
$\mathrm{CNF}^{2}$ & 6,78 & 63,89 & 35,71 & 35,39 & 35,08 & 34,71 \\
$\mathrm{CT}^{2}$ & 85,13 & 86,75 & 79,86 & 80,06 & 80,26 & 80,42 \\
\hline
\end{tabular}

${ }^{1} \% ;{ }^{2} \% \mathrm{MS} ;{ }^{3} \mathrm{kcal} / \mathrm{g} \mathrm{MS} ; \mathrm{MS}$ - matéria seca, MO - matéria orgânica, MM - matéria mineral, PB - proteína bruta, FDN - fibra em detergente neutro, FDA - fibra em detergente ácido, EE - extrato etéreo, EB - energia bruta, CNF carboidratos não fibrosos, $\mathrm{CT}$ - carboidratos totais.

A planilha de cálculo para a determinação da viabilidade econômica foi montada com base nos custos em reais por $\mathrm{kg}$ de peso vivo $(\mathrm{R} \$ / \mathrm{kgPV})$, do ganho de peso $(\mathrm{kg})$ dos animais/tratamento, do custo da ração total ( $\mathrm{R} \$ / \mathrm{kg})$, dos custos de obtenção do farelo de manga, do volumoso e concentrado e do consumo total de ração por tratamento. A planilha foi montada com base nos conceitos de benefício líquido e de taxa marginal de retorno (La formulación..., 1988) para cálculo da taxa de retorno em relação aos custos das rações experimentais.

O experimento foi realizado em delineamento inteiramente ao acaso, com seis repetições. Contrastes ortogonais foram utilizados para avaliar os efeitos de ordem linear e quadrático da porcentagem do farelo de manga em substituição ao milho. Os procedimentos estatísticos foram realizados adotando-se 5\% de significância. 


\section{Pereira et al.}

\section{RESULTADOS E DISCUSSÃO}

Não houve efeito da inclusão do farelo de manga ( $\mathrm{P}>0,05)$ sobre o consumo de matéria seca (CMS) expresso em g/dia e \% de peso vivo (Tab. 3), o que provavelmente está relacionado às semelhanças entre as dietas avaliadas isonitrogenadas. $\mathrm{O}$ consumo variou de $1156 \mathrm{~g} / \mathrm{dia}$, ou $3,7 \%$ do PV, para o tratamento com $33 \%$ de substituição, a $1297 \mathrm{~g} /$ dia, ou $4,9 \%$ do PV, para o tratamento com $66 \%$ de substituição.

Barroso et al. (2006), ao avaliarem o desempenho de ovinos confinados, com $23 \mathrm{~kg}$ de peso vivo médio inicial, alimentados com resíduo de vitivinícola associado a diferentes fontes energéticas, observaram valor médio de CMS em g/dia igual a 1166, valor inferior ao encontrado no presente trabalho. Parente et al. (2009), ao estudarem o desempenho de ovinos confinados, com peso vivo inicial médio igual a $25 \mathrm{~kg}$, submetidos a dietas à base de feno de tifton 85 com $19 \%$ de resíduo de caju ou maracujá, observaram valor médio de CMS igual a $1557 \mathrm{~g} / \mathrm{dia}$, valor mais alto que o obtido no presente trabalho. Segundo os autores, esse alto valor de CMS observado pode ser explicado pelo fato de o concentrado e o volumoso utilizados estarem finamente moídos, resultando em maior taxa de passagem.

Tabela 3. Consumo de matéria seca (CMS), de proteína bruta (CPB), de fibra em detergente neutro (CFDN) e de fibra em detergente ácido (CFDA) por ovinos, em função da porcentagem de inclusão de farelo de manga

\begin{tabular}{|c|c|c|c|c|c|c|c|}
\hline \multirow{2}{*}{ Variável } & \multicolumn{6}{|c|}{ Inclusão de farelo de manga (\%) } & \multirow{2}{*}{$\mathrm{CV}(\%)$} \\
\hline & 0 & 33 & 66 & 100 & $\mathrm{~L}$ & $\mathrm{Q}$ & \\
\hline \multicolumn{8}{|c|}{ g/dia } \\
\hline CMS & 1263 & 1156 & 1297 & 1218 & ns & $\mathrm{ns}$ & 18,37 \\
\hline $\mathrm{CPB}$ & 147,6937 & 139,3442 & 157,7776 & 152,3134 & ns & $\mathrm{ns}$ & 18,57 \\
\hline CFDN & 557,6663 & 518,1769 & 590,0331 & 562,645 & ns & $\mathrm{ns}$ & 18,52 \\
\hline CFDA & 343,385 & 329,905 & 387,3951 & 380,8675 & ns & $\mathrm{ns}$ & 18,84 \\
\hline \multicolumn{8}{|c|}{$\%$ Peso vivo } \\
\hline CMS & 4,17 & 3,66 & 4,93 & 4,12 & ns & $\mathrm{ns}$ & 25,15 \\
\hline CFDN & 1,84 & 1,64 & 2,24 & 1,9 & ns & ns & 25,39 \\
\hline
\end{tabular}

CV - coeficiente de variação; L - linear; Q - quadrático.

Não foram observadas variações $(\mathrm{P}>0,05)$ no CPB, CFDN e CFDA (Tab. 3), o que pode ser explicado pela semelhança no CMS apresentado e também pela similaridade nutricional entre as dietas avaliadas. Para CPB, CFDN e CFDA, os valores médios observados foram iguais a 149 , 557 e $360 \mathrm{~g} /$ dia.

Dantas Filho et al. (2007), ao estudarem o desempenho de ovinos Santa Inês alimentados com dietas à base de feno de tifton 85 com cinco porcentagens de inclusão de polpa de caju desidratada - 0,10,20,30 e 40\% -, observaram valores médios de CPB e CFDN iguais a 224 e $704 \mathrm{~g} / \mathrm{dia}$, mais alto que os observados no presente trabalho. Segundo esses autores, a inclusão da polpa de caju não influenciou o CPB, entretanto provocou aumento linear no CFDN

Rêgo et al. (2010), ao avaliarem o consumo de nutrientes de ovinos alimentados com silagem de capim-elefante com cinco porcentagens de adição - 0, 4, 8, 12 e $16 \%$ - de subproduto do processamento de manga desidratado, observaram que a adição de resíduo do processamento de manga não influenciou os consumos de MS, PB, assim como observado no presente trabalho, entretanto ocasionou reduções no consumo de FDN e de FDA, resultado diferente do observado no presente estudo.

Não houve efeito da inclusão do farelo de manga $(\mathrm{P}>0,05)$ sobre o GPD e o GPT, sendo que o ganho médio de peso médio observado de $176 \mathrm{~g} / \mathrm{dia}$, valor mais baixo que o encontrado por Parente et al. (2009), que obtiveram GPD médio igual a 198 g/dia, ao avaliarem o desempenho de ovinos Santa Inês submetidos a dietas à base de feno de tifton 85 com $19 \%$ de resíduo de caju ou maracujá. Entretanto, o valor obtido no presente estudo foi mais alto que o observado por Barroso et al. (2006), que obtiveram valor médio de GPD de $107 \mathrm{~g} / \mathrm{dia}$, ao avaliarem o resíduo de vitivinícola associado a diferentes fontes energéticas. 
Desempenho produtivo de ovinos...

Tabela 4. Ganho de peso diário (GPD), ganho de peso total (GPT) e conversão alimentar (CA) em ovinos alimentados com diferentes níveis de inclusão de farelo de manga na dieta

\begin{tabular}{lccccccc}
\hline \multirow{2}{*}{ Variável } & \multicolumn{9}{c}{ Inclusão de farelo de manga $(\%)$} & \multirow{2}{*}{ CV (\%) } \\
\cline { 2 - 6 } & 0 & 33 & 66 & 100 & L & Q & \\
\hline PVI (kg) & 22,37 & 23,33 & 23,83 & 23,67 & & & \\
PVF(kg) & 37,68 & 35,35 & 36,47 & 36,73 & & & \\
GPD & 204,22 & 160,22 & 168,44 & 174,22 & ns & ns & 23,45 \\
GPT & 15,32 & 12,02 & 12,63 & 13,07 & ns & ns & 23,45 \\
CA (kgMs/kgGPV) & 6,28 & 7,44 & 8,29 & 7,18 & ns & $*$ & 17,73 \\
\hline
\end{tabular}

PVI - peso vivo inicial, PVF - peso vivo final, CV - coeficiente de variação, L - linear, Q - quadrático.

${ }^{*} \mathrm{Y}=6,19628155+0,006183440-0,00051181 \mathrm{x}^{2}\left(\mathrm{r}^{2}=0,94\right)$.

Segundo Mertens (1994), 60 a 90\% das diferenças no desempenho animal estão diretamente relacionadas ao consumo. O CMS e os nutrientes apresentados no presente trabalho foram semelhantes, o que pode explicar a similaridade entre os ganhos de peso observados.

Verificou-se efeito quadrático $(\mathrm{P}<0,05)$ da porcentagem do farelo de manga sobre a CA (Tab. 4). Nos tratamentos em que o farelo de manga representou 33 ou $66 \%$ do concentrado, os valores de CA foram maiores, ou seja, a eficiência animal foi prejudicada, entretanto, no tratamento com 0 ou $100 \%$ de farelo de manga, observaram-se os menores valores de CA. Esse fato pode estar relacionado à presença de efeito associativo negativo, contudo o consumo de nutrientes e o ganho de peso não diferiram, indicando a necessidade da realização de outros estudos que melhor expliquem a natureza desse tipo de interação.

O valor de CA para o nível de $100 \%$ de inclusão do farelo de manga em substituição ao milho moído foi de $7,18 \mathrm{kgMS} / \mathrm{kgGPV}$, valor semelhante ao encontrado por Ferreira et al. (2009) quando avaliaram a CA de ovinos SPRD (sem padrão racial definido), com $20 \mathrm{~kg}$ de peso médio inicial, alimentados com silagem de capim-elefante contendo $10,5 \%$ do subproduto do pseudofruto do caju, entretanto foi superior aos obtidos pelos mesmo autores quando avaliaram a CA de ovinos alimentados com silagem de capim-elefante contendo $7 \%$ de subproduto de acerola.

Quanto à avaliação econômica, todas as dietas apresentaram benefícios líquidos e taxa de retorno sobre os custos diferenciados positivos (Tab. 5).

O tratamento com $33 \%$ de substituição apresentou o menor valor de taxa de retorno e benefício líquido, apesar do custo por quilograma da ração deste tratamento ser menor que a do tratamento controle, o que é atribuído ao menor ganho de peso observado nesse tratamento.

Tabela 5. Benefício líquido e taxa de retorno sobre o custo total das dietas de ovinos, em função dos níveis de inclusão do farelo de manga em substituição ao milho moído

\begin{tabular}{lcccc}
\hline \multirow{2}{*}{ Variável } & \multicolumn{4}{c}{ Inclusão de farelo de manga (\%) } \\
\cline { 2 - 5 } & 0 & 33 & 66 & 100 \\
\hline R\$/kg PV & 4,00 & 4,00 & 4,00 & 4,00 \\
GP/ Tratamento (kg) & 91,90 & 72,10 & 75,80 & 78,40 \\
Valor total PV (R\$) [A] & 367,60 & 288,40 & 303,20 & 313,60 \\
& & & & 0,30 \\
R\$/Kg de ração & 0,50 & 0,44 & 0,37 & 555,33 \\
Consumo de dieta total (kg) & 576,02 & 527,09 & 591,36 & 167,83 \\
Custo da dieta total (R\$) [B] & 290,07 & 230,39 & 218,78 & 145,77 \\
& & & & 86,85 \\
Benefício líquido (R\$) [C=A-B] & 77,53 & 58,01 & 84,42 & 38,59 \\
Taxa de retorno (\%) [C/B x 100] & 26,73 & 25,18 & & \\
\hline PV - pes
\end{tabular}

PV - peso vivo, GP - ganho de peso. 
O tratamento com $100 \%$ de substituição ao milho, seguido pelo tratamento com $66 \%$ de substituição, apresentou as maiores taxas de retorno e benefício líquido. A margem de diferença favorável ao tratamento com $100 \%$ de substituição se deve à diferença do custo total por quilograma de ração entre os tratamentos com 0 e $100 \%$ de inclusão do farelo de manga em substituição ao milho moído.

\section{CONCLUSÕES}

O farelo de manga pode substituir o milho em até $100 \%$ em dietas de ovinos confinados, sem interferir no consumo e no ganho de peso. A substituição do milho por farelo de manga pode reduzir o custo com alimentação e aumentar o benefício líquido e a taxa de retorno sobre o custo total das dietas de ovinos confinados. O farelo de manga é um alimento alternativo, podendo constituir a base do concentrado energético de ovinos em confinamento.

\section{REFERÊNCIAS}

ASSOCIATION of official agriculture chemists - AOAC. Official methods of analyses of the Association of Agriculture Chemists. 13.ed. Washington: AOAC, 1980. 1015p.

AZEVEDO, J.A.G.; VALADARES FILHO, S.C.; PINA, D.S. et al. Nutritional diversity of agricultural and agro-industrial by-products for ruminant feeding. Arq. Bras. Med. Vet. Zootec., v.64, p.1246-1255, 2012.

BARROSO, D.D.; ARAÚJO, G.G.L.; SILVA, D.S. et al. Desempenho de ovinos terminados em confinamento com resíduo desidratado de vitivinícolas associado a diferentes fontes energéticas. Cienc. Rural, v.36, p.1553-1557, 2006.

DANTAS FILHO, L.A.; LOPES, J.B.; VASCONCELOS, V.R. et al. Inclusão de polpa de caju desidratada na alimentação de ovinos: desempenho, digestibilidade e balanço de nitrogênio. Rev. Bras. Zootec., v.36, p.147-154, 2007.
FERREIRA, A.C.H.; NEIVA, J.N.M.; RODRIGUEZ, N.M. et al. Desempenho produtivo de ovinos alimentados com silagens de capim-elefante contendo subprodutos do processamento de frutas. Rev. Cienc. Agron., v.40, p.315-322, 2009.

HALL, M.B. Neutral detergent-soluble carbohydrates. Nutritional relevance and analysis. Florida: University of Florida, 2000. (Bulletin, 339)

INSTITUTO Brasileiro de Geografia e Estatística - IBGE. Produção agrícola municipal (PAM): quantidade produzida. Disponível em: <http://www.sidra.ibge.gov.br/bda/pesquisas/pa m/ default.asp>. Acessado em: 29 abr. 2011.

LA FORMULACIÓN de recomendaciones a partir de datos agronómicos: un manual metodológico de evaluación económica. México: CIMMYT, 1988. 79p.

LAGE, J.F.; RODRIGUES, P.V.; PEREIRA, L.G.R. et al. Glicerina bruta na dieta de cordeiros terminados em confinamento. Pesq. Agropec. Bras., v.45, p.1012-1020, 2010.

MERTENS, D.R. Regulation of forage intake. In: FAHEY, J.R., GEORGE, C. (Ed). Forage quality, evaluation and utilization. Madison: American Society of Agronomy, 1994. p.450493.

PARENTE, H.N.; MACHADO, T.M.M.; CARVALHO, F.C. et al. Desempenho produtivo de ovinos em confinamento alimentados com diferentes dietas. Arq. Bras. Med. Vet. Zootec., v.61, p.460-466, 2009.

RÊGO, M.M.T.; NEIVA, J.N.M.; REGO, A.C. et al. Intake, nutrients digestibility and nitrogen balance of elephant grass silages with mango byproduct addition. Rev. Bras. Zootec., v.39, p.74$80,2010$.

SILVA, D.J.; QUEIROZ, A.C. Análise de alimentos: métodos químicos e biológicos. 3.ed. Viçosa: UFV, 2002. 235p.

VAN SOEST, P.J.; ROBERTSON, J.B.; LEWIS, B.A. Methods for extraction fiber, neutral detergent fiber and non-starch polysaccarides in relation to animal nutrition cows. J. Dairy Sci., v.74, p.3583-3597, 1991. 